Age Dating of SRM U050: LLNL Results

R. W. Williams, A. M. Gaffney, K. C. Schorzman, A. C. Villa

August 26, 2013 
This document was prepared as an account of work sponsored by an agency of the United States government. Neither the United States government nor Lawrence Livermore National Security, LLC, nor any of their employees makes any warranty, expressed or implied, or assumes any legal liability or responsibility for the accuracy, completeness, or usefulness of any information, apparatus, product, or process disclosed, or represents that its use would not infringe privately owned rights. Reference herein to any specific commercial product, process, or service by trade name, trademark, manufacturer, or otherwise does not necessarily constitute or imply its endorsement, recommendation, or favoring by the United States government or Lawrence Livermore National Security, LLC. The views and opinions of authors expressed herein do not necessarily state or reflect those of the United States government or Lawrence Livermore National Security, LLC, and shall not be used for advertising or product endorsement purposes.

This work performed under the auspices of the U.S. Department of Energy by Lawrence Livermore National Laboratory under Contract DE-AC52-07NA27344. 


\section{Age Dating of SRM U050: LLNL Results}

\section{Ross Williams, Amy Gaffney, Kerri Schorzman, Adam Villa}

Two solutions of U050 standard (\#1 and \#2) were prepared at LLNL from two separate bottles of the SRM. These solutions were analyzed for ${ }^{230} \mathrm{Th}$ and ${ }^{234} \mathrm{U}$ by the methods described in the procedures supplied previously, with some modification of the Th purification procedure that will be described in our presentation.

The first step is to measure the uranium concentration and isotopic composition of the primary solutions. To accomplish this, it is necessary to prepare secondary and sometimes tertiary dilutions to lower the $\mathrm{U}$ concentration to a range where conservative amounts of ${ }^{233} \mathrm{U}$ spike can be used. One twostep dilution was made in January, and a replicate independent two-step dilution was made in February 2013. The results of these $U$ concentration measurements did not agree very well, so a third set of single-step dilutions were made, spiked, and analyzed in July 2013. These three measurements are shown in the following figures.

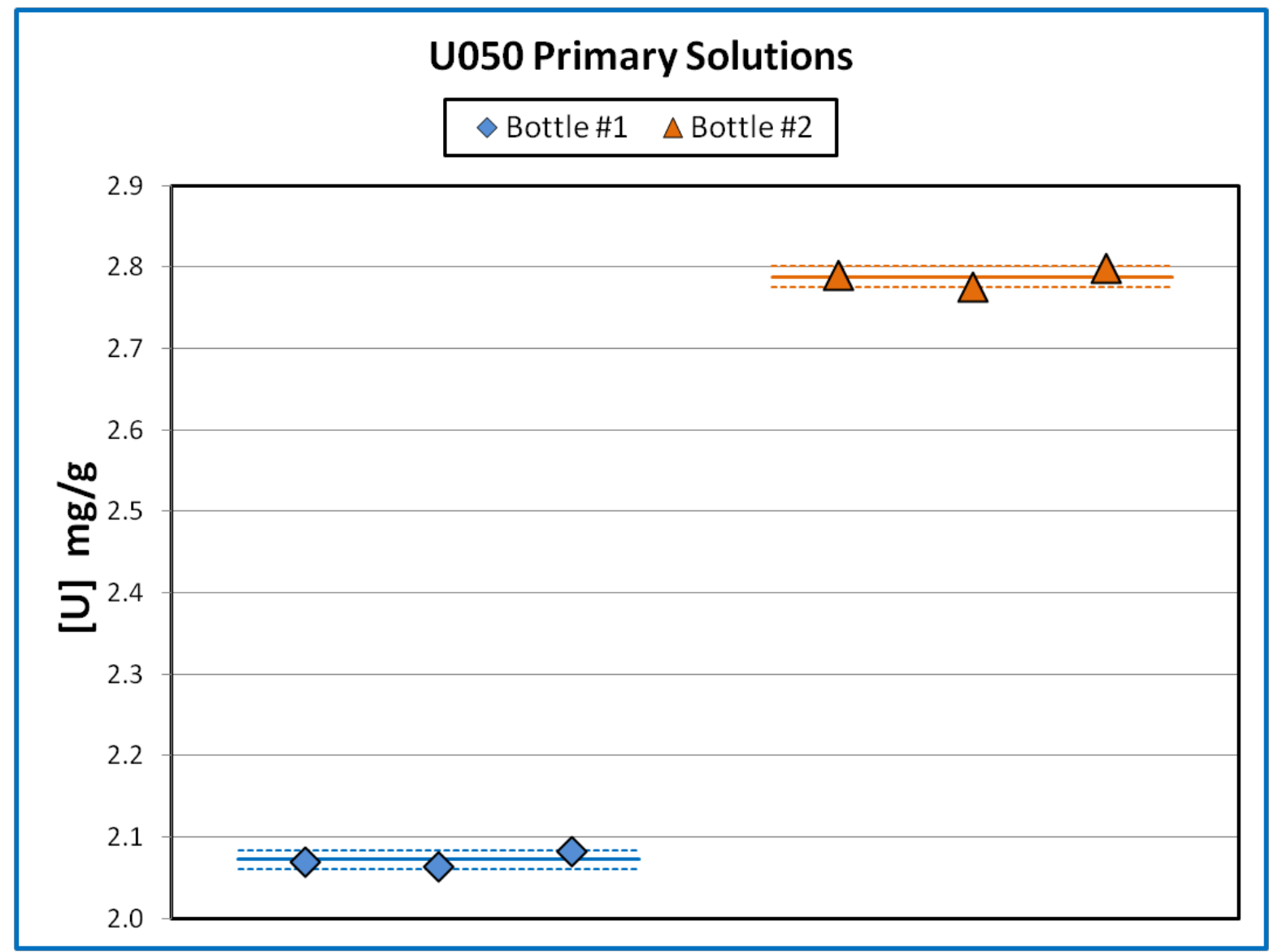

Fig. 1 The uranium concentration measurements of the primary solutions. The mean and $2 X$ the standard error $(n=3)$ is shown by the solid and dashed lines for each primary solution. 


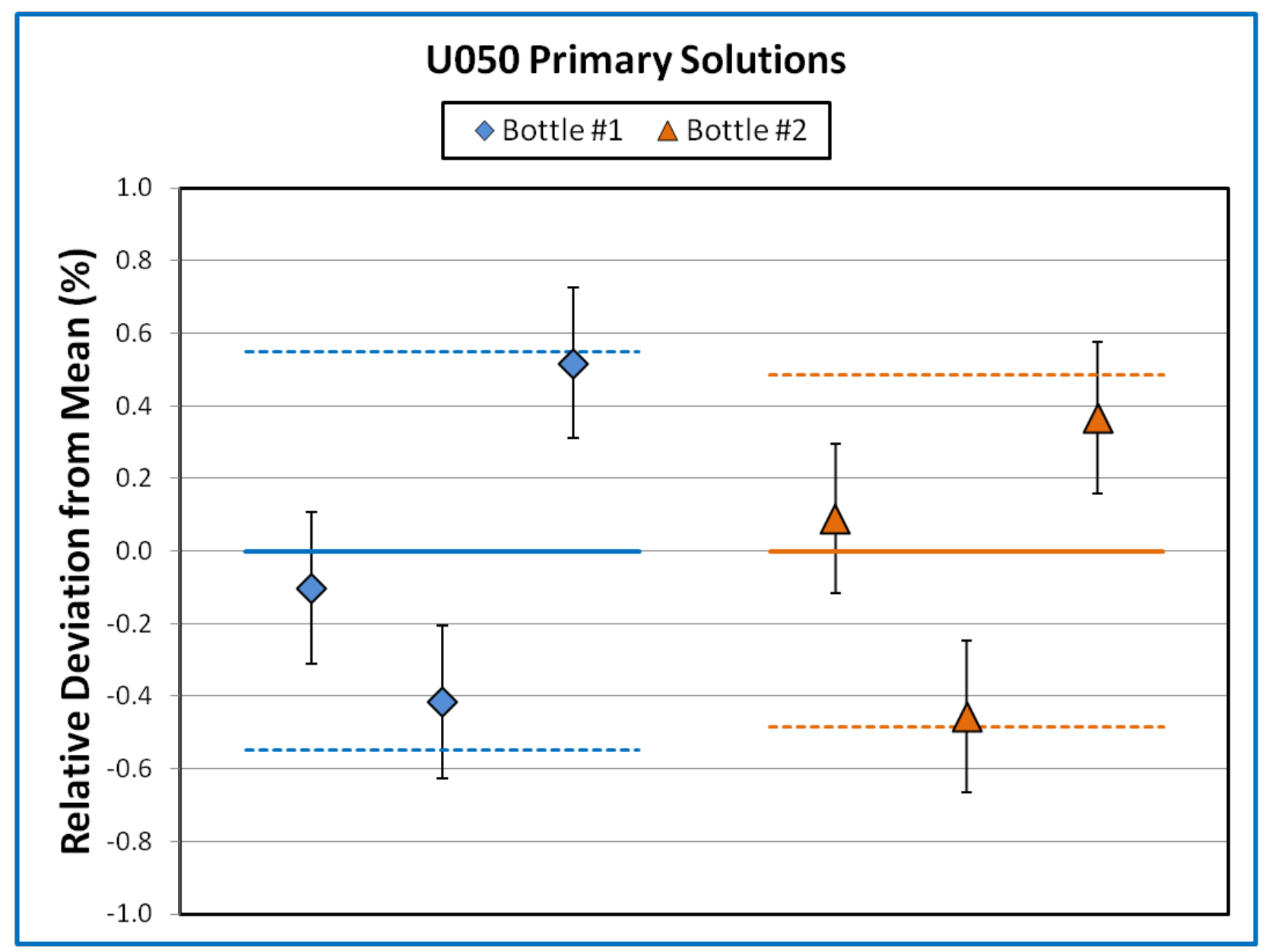

Fig. 2 The uranium results plotted as relative deviation from the mean. The error bars on the individual analyses are expanded uncertainties $(k=2)$.

The expanded uncertainties on the individual analyses include all known sources of uncertainty. They include the uncertainties from: weighing the samples, the dilution solutions, and the ${ }^{233} \mathrm{U}$ spike; the calibration of the spike; the mass spectrometry measurements; and the uncertainty on the certified value of the standard used for mass bias correction and gain calibration. These expanded uncertainties are only about $0.2 \%$, but the range of the replicates is almost $1 \%$. From these results, we conclude that there is additional uncertainty in this method that we do not currently understand, and we welcome any input from our colleagues to improve our understanding, or suggestions for improvement of this method.

For the calculation of ages of U050 reported here, we use the average ${ }^{234} \mathrm{U}$ value for the primary solutions obtained from these three $U$ analyses, and the larger uncertainties $(0.5-0.6 \%, k=2)$ associated with these averages. Four independent analyses of ${ }^{230} \mathrm{Th}$ in the primary solutions were made.

The results of these age-dating analyses are given in the following table: 


\begin{tabular}{|l|r|r|r|r|r|r|}
\hline Sample ID & $\begin{array}{r}\text { Reference } \\
\text { Date }\end{array}$ & $\begin{array}{r}\text { 230Th-234U } \\
\text { Model Age } \\
\text { (years) }\end{array}$ & $\begin{array}{r}\text { expanded } \\
\text { combined } \\
\text { std. uncert. }\end{array}$ & $\begin{array}{r}\text { Model } \\
\text { (k=2) }\end{array}$ & $\begin{array}{r}\text { Expanded } \\
\text { Uncertainty } \\
\text { (days) }\end{array}$ \\
\hline U050 \#1 & 27-Mar-13 & 55.965 & 0.217 & 0.434 & 8-Apr-57 & 158.6 \\
\hline U050 \#1 & 4-Apr-13 & 56.190 & 0.219 & 0.439 & 24-Jan-57 & 160.3 \\
\hline U050 \#1 & 22-Jul-13 & 56.327 & 0.216 & 0.433 & 24-Mar-57 & 158.1 \\
\hline U050 \#1 & 22-Jul-13 & 56.358 & 0.217 & 0.434 & 13-Mar-57 & 158.6 \\
\hline & & & & & & \\
\hline U050 \#2 & 27-Mar-13 & 56.113 & 0.206 & 0.412 & 13-Feb-57 & 150.4 \\
\hline U050 \#2 & 4-Apr-13 & 56.197 & 0.210 & 0.420 & 21-Jan-57 & 153.5 \\
\hline U050 \#2 & 22-Jul-13 & 56.300 & 0.204 & 0.407 & 3-Apr-57 & 148.8 \\
\hline U050 \#2 & 22-Jul-13 & 56.350 & 0.204 & 0.407 & 16-Mar-57 & 148.7 \\
\hline
\end{tabular}

These results give an average model date and uncertainty of 5-March-1957 \pm 30 days. The uncertainty is the standard deviation of the population $(n=8)$. The LLNL results are plotted in the following figure, along with a "paper age" of 11-Jun-1958.

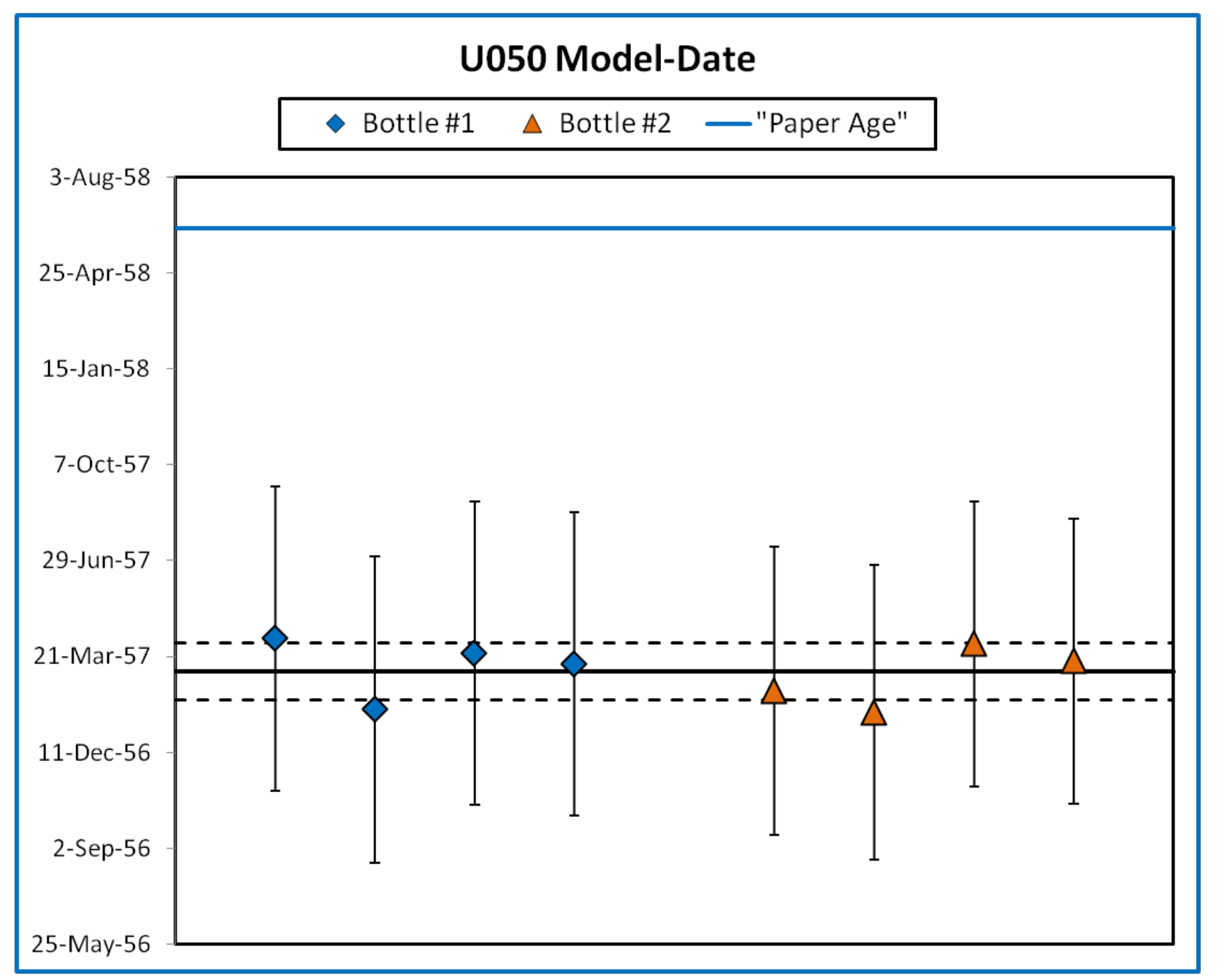

U050 Age Dating: LLNL 
A representative uncertainty budget for a single analysis with an expanded uncertainty of 150 days is given below.

\begin{tabular}{|l|r|}
\hline \multicolumn{2}{|c|}{$\begin{array}{c}\text { Single analysis } \\
\text { Simplified Uncertainty Budget }\end{array}$} \\
\hline 230Th measurement & $45.97 \%$ \\
\hline 234U measurement & $46.97 \%$ \\
\hline 230Th half-life & $0.00 \%$ \\
\hline 234U half-life & $7.06 \%$ \\
\hline \multicolumn{2}{|c|}{$100 \%$} \\
\hline
\end{tabular}

The information that we have for the age of this material is obtained from two independent reviews of documents associated with its production, but these reviews are not consistent with each other. This information follows:

\begin{tabular}{|c|r|r|}
\hline Review & From Cascade & Purification \\
\hline 1 & 4-Oct-57 & 7-Oct to 7-Nov-1958 \\
\hline & Date Received at & Kate Purification \\
K25 & Finished \\
\hline 2 & $21-$ Mar-58 & 11-Jun-58 \\
\hline
\end{tabular}

The highlighted date (4-Oct-1957) is very close to the earliest date that is in these reviews (12-Sep-1957) for the retrieval of materials from the Oak Ridge cascade that were used to produce the NBS SRMs.

Review number 2 notes that: "These are the dates obtained from the K25 documentation obtained both from the New Brunswick Laboratory and trough ( sic) the NIST library from the DOE/K25 archives of at Oak Ridge. The dates given in the documents are not necessarily the date of "system closure" for the material that went into each of the SRMs. Although care was taken to identify each of the SRM's from the documentation it should be noted that no where (sic) in the documentation is a particular material, or preparation and date directly associated with something called "UXXX". Therefore, the associations are done by best comparisons with the isotopic compositions."

This statement serves to illustrate the point that there is a great deal of uncertainty in the "paper age" of these materials.

The LLNL results above, taken at face value, indicate that $U 050$ either contained excess ${ }^{230} \mathrm{Th}$ at the time it came off the cascade, and was never purified, or, that the purification process, whatever it was, either contaminated the material with ${ }^{230} \mathrm{Th}$ or did not completely remove whatever ${ }^{230} \mathrm{Th}$ may have been present when it came off the cascade. 